\title{
Long-term results of conjoined unification venoplasty for multiple portal vein branches of the right liver graft in living donor liver transplantations
}

\author{
Sung Yeon Yoo', Shin Hwang ${ }^{1}$, Tae-Yong Ha ${ }^{1}$, Gi-Won Song ${ }^{1}$, Dong-Hwan Jung ${ }^{1}$, Gil-Chun Park ${ }^{1}$, \\ Chul-Soo Ahn', Deok-Bog Moon', Ki-Hun Kim', Young-In Yoon', Yo-Han Park², Hui-Dong Cho', \\ Yong-Kyu Chung ${ }^{1}$, Sang-Hyun Kang ${ }^{1}$, Jin-Uk Choi ${ }^{1}$, Sung-Gyu Lee ${ }^{1}$ \\ ${ }^{1}$ Department of Surgery, Asan Medical Center, University of Ulsan College of Medicine, Seoul, Korea \\ ${ }^{2}$ Department of Surgery, Busan Paik Hospital, Inje University College of Medicine, Busan, Korea
}

Background: Autologous portal vein Y-graft (PYG) interposition has been the standard procedure for reconstruction of double portal vein (PV) orifices of right liver grafts during living donor liver transplantations. However, it has the disadvantage of being vulnerable to anastomotic stenosis. A refined technique of conjoined unification venoplasty (CUV) was developed to secure PV reconstruction.

Methods: We reviewed the surgical outcomes in PV reconstructions using CUVs in 21 cases which were followed up for $>3$ years.

Results: The mean age of recipients was $51.7 \pm 4.9$ years. The model for end-stage liver disease score was 15. 3 \pm 6.4 . The graft-recipient weight ratio was $1.12 \pm 0.21$. Recipient PYGs were harvested in all cases. All living donors were blood relatives or relatives through marriage with type III PV anomalies. The number of right liver graft PV orifices was two in 19 cases and three in two cases. For the central intervening vein patch, a PV segment was used in six cases, and an autologous greater saphenous vein patch was used in the remaining 15 cases. The 21 patient cohort displayed a 100\% 4-year patient survival rate. None of them underwent any PV interventions including interventional stenting. Serial follow-up computed tomography scans revealed that the reconstructed PV showed early reshaping with a stable streamlined configuration for over 3 years. Conclusions: PV reconstruction using the CUV technique appears to be significantly more effective in preventing PV complications. We believe that CUV is a useful technique to reconstruct right liver grafts with multiple PV orifices.

Keywords: Living donor liver transplantation; Y-graft; Portal vein anomaly; Anastomotic stenosis

\section{INTRODUCTION}

Anomalous portal vein (PV) branching of the right liver is present in a considerable number of living liver donors

Received July 28, 2019

Revised September 30, 2019

Accepted October 1, 2019

Correspondence to: Shin Hwang

Department of Surgery, Asan Medical Center, University of Ulsan College of Medicine, 88 Olympic-ro 43-gil, Songpa-gu, Seoul 05505, Korea

Tel: +82-2-3010-3930, Fax: +82-2-3010-6701

E-mail: shwang@amc.seoul.kr
[1]. It becomes important in living donor liver transplantations (LDLTs) because it usually results in double PV orifices at the right liver grafts. These double PV orifice grafts require unification procedures because their direct reconstruction using one-to-one anastomoses are not practical. To unify double PV orifices, several reconstructive methods have been developed. Autologous portal vein Y-graft (PYG) interposition has been regarded as the standard procedure [2-6]. However, autologous PYG interpositions occasionally result in stenosis from excessive redundancy of the right posterior section

This is an Open Access article distributed under the terms of the Creative Commons Attribution Non-Commercial License (http://creativecommons. org/ licenses/by-nc/4.0/) which permits unrestricted non-commercial use, distribution, and reproduction in any medium, provided the original work is properly cited. 


\section{HIGHLIGHTS}

- Portal vein reconstruction using the conjoined unification venoplasty technique appears to be effective in preventing portal vein complications.

- It is a useful technique to reconstruct right liver grafts with multiple portal vein orifices.

of the PV branch. To resolve this issue, we developed a conjoined unification venoplasty (CUV) method which is a technical modification of conventional autologous PYG interpositions $[7,8]$. This study presents the long-term outcomes of CUVs for reconstructions of double PV orifice grafts in a high-volume LDLT center.

\section{METHODS}

\section{Study Design}

This was a retrospective single-arm study regarding the outcomes of CUV for reconstruction of double PV orifice grafts. The primary purpose of this study was to present the 3-year actual patency rates of PV reconstructions using CUV. The secondary purpose was to present the long-term morphometric changes in PV reconstructions using CUVs.

CUVs were first applied to LDLTs in July 2014 at Asan Medical Center. Therefore, the study period was set to
24 months from July 2014 to December 2015. During this study period, 21 patients were selected. Recipients showing hepatocellular recurrence were excluded to avoid unnecessary bias. The participants underwent regular follow-ups at our institution's outpatient clinic. We collected follow-up data until December 2018 or until their deaths making the observation period $>3$ years. This study protocol was approved by the Institutional Review Board of our institution (IRB No. 2018-1386).

\section{Surgical Techniques of CUV}

The surgical techniques for CUV included excising a $5-\mathrm{mm}$-long segment of the autologous sectional PV branch creating a $5 \times 12-\mathrm{mm}$-sized venous patch. When a PV patch was not available, an autologous greater saphenous venous patch was used. This patch was inserted as a central intervening venous patch between the two sectional PV orifices with small niches. Crutch-opened autologous PYGs were anastomosed to the unified PV orifice grafts creating a potbelly-shaped PV confluence (Fig. 1). Details of these procedures have been presented elsewhere $[7,8]$.

\section{Morphometric Evaluations of PV Reconstructions by CUVs}

According to our LDLT management protocol, Doppler ultrasonography was performed each day during the first
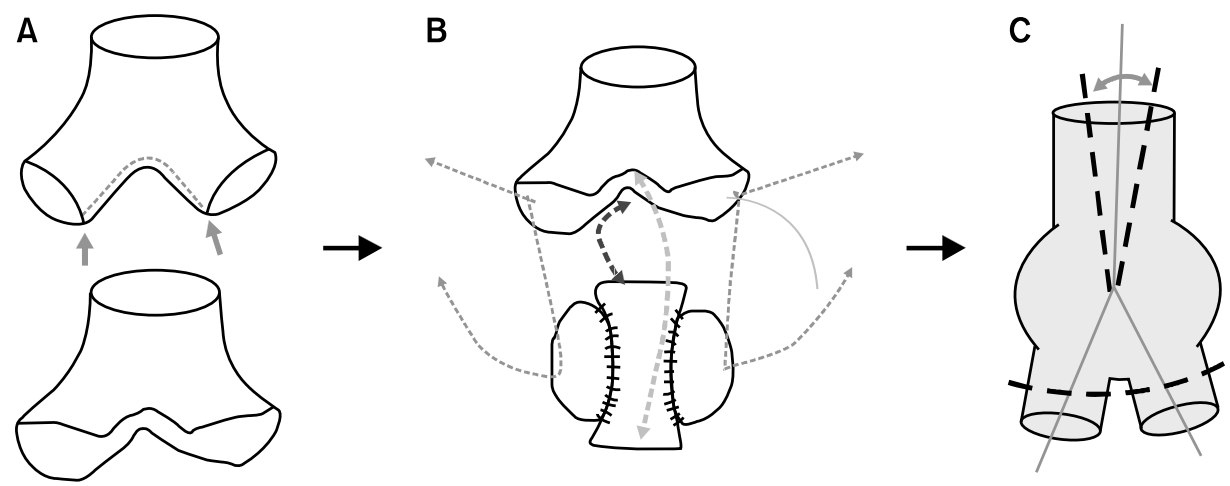

Fig. 1. Illustration of the conjoined unification venoplasty technique. (A) First, the recipient's autologous portal vein (PV) Y-graft was harvested and the crutch of the PV Y-graft was opened (arrows) creating a funnel-shaped vessel graft. (B) A central vein patch is attached between the two sectional PV orifices of the right liver graft, which converts the two PV orifices to one large PV orifice. The crutch-opened PV graft is anastomosed to the unified PV graft. Dotted lines indicate the approximation points for end-to-end anastomosis. (C) The final configuration of the unified PV makes a potbelly-shaped PV confluence. This conjoined PV confluence provides a wider range of tolerance for malalignment (bidirectional arrow) and PV size mismatches. 
week after LDLT, and once or twice per week thereafter during hospitalization. Post-transplant dynamic computed tomography (CT) scans were routinely performed every week while the patients were in the hospital, and at $1,3,6$, and 12 months after LDLTs. Thereafter, follow-up abdominal CT scans were repeated annually for 5 years and biannually after 5 years. In this study, we reconstructed the shape of the PV system using portal phase CT images in a three-dimensional mode and compared their serial changes for the first 3 years after LDLTs.

\section{Statistical Analysis}

All numerical data were presented as mean values with standard deviations. The patency rates were determined using the Kaplan-Meier method. Statistical analyses were performed using IBM SPSS ver. 22. 0 (IBM Corp., Armonk, NY, USA).

\section{RESULTS}

\section{Patient Profiles}

The clinical profiles of 21 patients who underwent LDLTs using a right liver graft were as follows: mean age, $51.7 \pm 4.9$ years; male to female ratio, $15: 6$; primary diagnoses: hepatitis $\mathrm{B}$ viral infections in 14 patients, hepatitis $\mathrm{C}$ viral infections in two patients, alcoholic liver disease in three patients, and other diseases in two pa- tients; model for end-stage liver disease scores, 15. $3 \pm$ 6. 4; ABO blood-incompatible LDLTs, 2; and graft-recipient weight ratio, $1.12 \pm 0.21$.

Recipient PYGs were harvested in all cases. For the central intervening vein patch, a PV segment was used in six cases and an autologous greater saphenous vein patch was used in the remaining 15 cases (Figs. 2 and 3).

All living donors were blood-relatives or relatives through marriage with type III PV anomalies, in which the right anterior and posterior PV branches were separated by a rectangular gap $[4,5]$. The number of right liver graft PV orifices was two in 19 cases and three in two cases. All of the PV orifices were unified into one orifice using the CUV technique.

\section{Long-Term Patency and Morphometric Changes of PV Reconstructions by CUV}

During a mean follow-up of 4 years, the 21 patient cohort displayed a $100 \%$ 4-year patient survival rate. None of them underwent any PV interventions including interventional stenting. Three-dimensional reconstruction of the recipient PVs using serial follow-up CT scans showed progressive streamlined reshaping (Fig. 3). The PV confluence portion was over-expanded at portal reperfusion; however, within a few weeks, it showed streamlined configuration with the entire reshaping process occurring over several months. Thereafter, the stable reshaped configuration of the reconstructed PV maintained for 3 years.

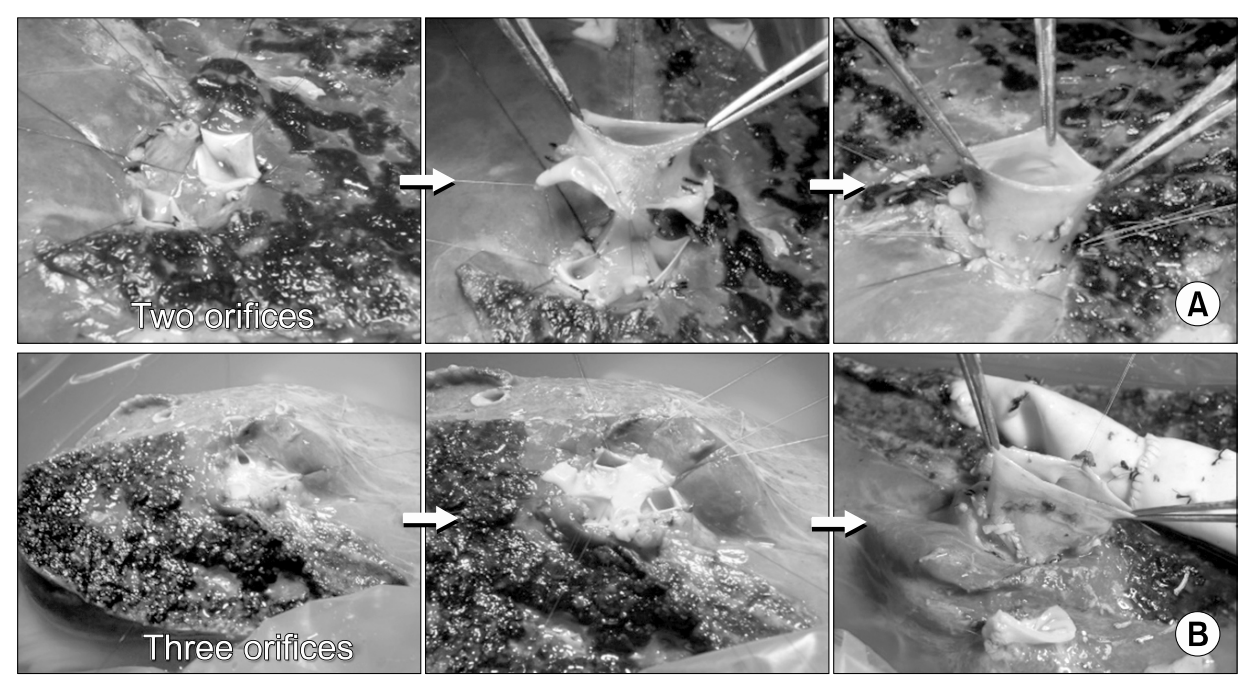

Fig. 2. Operative photographs of the conjoined unification venoplasty technique applied to the two and three portal vein (PV) orifices. (A) The two PV orifices were unified by a central vein patch from a short PV segment. The crutch-opened autologous PV Y-graft is anastomosed to make a single PV orifice. (B) The three $\mathrm{PV}$ orifices were unified by a Yshaped incised central vein patch from an autologous greater saphenous vein segment. The crutch-opened autologous PV Y-graft is attached to anastomose this enlarged $\mathrm{PV}$ graft orifice. 

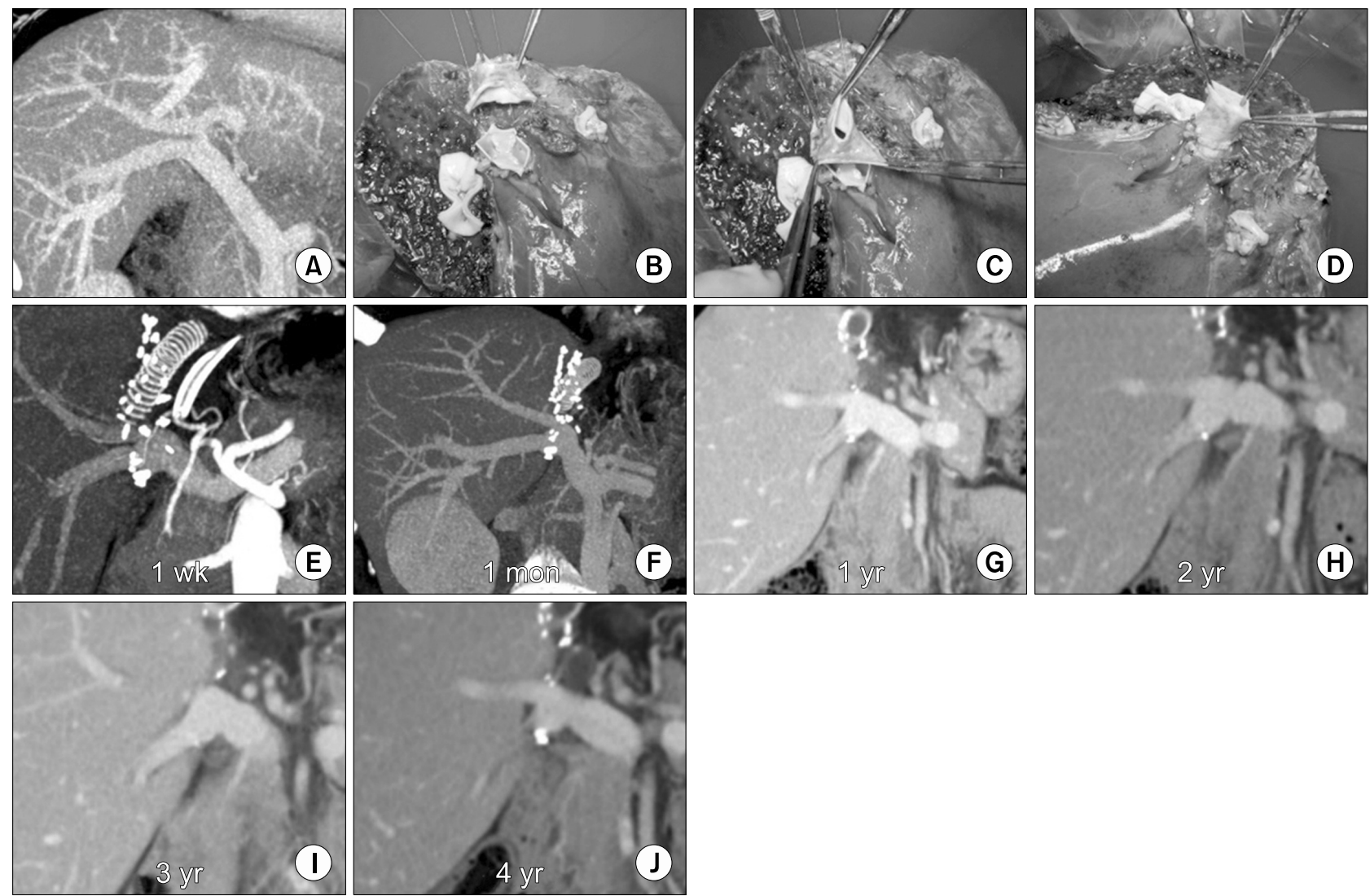

Fig. 3. Sequential changes of the recipient portal vein (PV) reconstructed by the conjoined unification venoplasty technique. (A) The donor PV showed a type III anomaly. (B-D) Two graft PV orifices were unified by a conjoined unification venoplasty. (E-J) Computed tomography portal phase follow-up images showed slight expansion of the anastomotic confluence during the first week, which was reshaped at 1 month. There was no noticeable configurational changes in the streamlined reshaped PV after 1 year.

\section{DISCUSSION}

Following its first introduction in 2001, an autologous PYG interposition has been the standard procedure for grafts with double PV orifices because it is technically simplistic and has excellent long-term outcomes [1,5]. An autologous PYG interposition is simple and intuitive. However, in practice, it is often technically demanding and has occasionally resulted in stenoses because of anatomical variations and discrepancies between the recipient and graft PVs. To resolve such issues, we developed a CUV method to improve the technique of conventional PYG interposition. Long-term outcomes of PV reconstructions by CUV were excellent and showed no complications for $>3$ years in this study.

In our institution, conventional autologous PYG interpositions and CUVs have been concurrently performed depending on the surgeons' preference and donor PV anatomies. Based on our experience, primary indications for CUVs appear to be a combination of one small and one large sectional PV orifice, the presence of a small accessory PV branch, a long extrahepatic PV branch, and the poor conditions of autologous PYGs. In cases with multiple PV grafts $(>2)$ or severely damaged recipient PYGs, CUV should be adopted preferentially because these conditions are highly disadvantageous if conventional autologous PYG interposition is considered [9]. Typically, we performed intraoperative cine-portograms to determine the shape of the reconstructed PV when PYG interpositions were undertaken. It also enabled us to detect and manage the spontaneous portosystemic collaterals [10]. We believe that CUVs or similar techniques are important components of standardized modified right lobe grafts in order to minimize vascular complications in 
adult LDLTs [11,12].

The only disadvantage of CUVs compared with conventional PYG interpositions is the complexity of the bench work. If surgeons fully understand the primary mechanisms of CUV reconstruction, the surgical design for CUV may not be difficult. The most critical technical point is to ensure that the PV confluence portion is sufficiently large, which automatically resolves anatomical variations and discrepancies between the recipient and graft PVs. The technical principles of CUV are markedly similar to those of the quilt venoplasty technique used for outflow vein reconstructions of the extended right lobe grafts $[13,14]$. PV reconstructions with CUVs do not require any anticoagulant or antiplatelet agents after LDLTs.

CUVs can be performed using vein sources other than autologous PYGs, such as long autologous greater saphenous venous patches or fresh cold-stored homologous venous patches. We do not recommend using cryopreserved venous grafts for PV replacements because they can induce aneurysmal dilatation or shrinkage [15].

The primary mechanism of CUV reconstruction is a gradual reshaping of the dual PVs into one single PV according to hemodynamic principles. Follow-up CT images revealed that the luminal configuration of the unified PV reconstruction with CUV was markedly similar to that obtained using a single PV reconstruction. This indicates that the PV confluence portion usually over-expands at the time of portal reperfusion, but shortly thereafter, reshapes and streamlines according to the principles of hemodynamics. This reshaping process began within a few weeks, stabilized within several months, and continued for $>3$ years. We expect that this reshaped configuration will continue to be stable over the life of the patient.

In conclusion, PV reconstructions using the CUV technique appear to be significantly effective in preventing PV complications. We believe that the CUV method is a useful technical option for reconstruction of right liver grafts with multiple PV orifice grafts during LDLTs because it can overcome the disadvantages of conventional autologous PYG interpositions.

\section{ACKNOWLEDGMENTS}

\section{Conflict of Interest}

No potential conflict of interest relevant to this article was reported.

\section{Funding/Support}

This study was supported by the intramural research fund of Asan Medical Center Organ Transplantation Center (2018-01).

This study was supported by research grant from the Korean Society for Transplantation (2019-04-01002005).

\section{ORCID}

Sung Yeon Yoo https://orcid. org/0000-0002-3145-9354

Shin Hwang https://orcid. org/0000-0002-9045-2531

Tae-Yong Ha https://orcid. org/0000-0001-9932-0212

Gi-Won Song https://orcid. org/0000-0002-4235-0434

Dong-Hwan Jung https://orcid. org/0000-0001-5984-023X

Gil-Chun Park https://orcid. org/0000-0003-1631-3258

Chul-Soo Ahn https://orcid. org/0000-0002-3844-3646

Deok-Bog Moon https://orcid. org/0000-0002-8209-3540

Ki-Hun Kim

https://orcid. org/0000-0002-4016-0995

Young-In Yoon

https://orcid. org/0000-0002-9308-0366

Yo-Han Park

https://orcid. org/0000-0002-2242-0968

Hui-Dong Cho

Yong-Kyu Chung

https://orcid. org/0000-0001-8501-3385

Sang-Hyun Kang

https://orcid. org/0000-0002-2132-2450

https://orcid, org/0000-0002-8518-1941

Jin-Uk Choi

https://orcid. org/0000-0001-8078-0593

Sung-Gyu Lee

\section{Author Contributions}

Conceptualization: SH. Data curation: SYY, TYH, GWS, DHJ, GCP, CSA, DBM, KHK, YIY, YHP, HDC, YKC, SHK, JUC. Formal analysis: SH, TYH. Funding acquisition: SH. Methodology: SH, DHJ. Project administration: SH, SGL. Visualization: SH. Writing - original draft: SH, SYY. Writing - review \& editing: SH.

\section{REFERENCES}

1. Hwang S, Lee SG, Lee YJ, Sung KB, Park KM, Kim 
$\mathrm{KH}$, et al. Lessons learned from 1,000 living donor liver transplantations in a single center: how to make living donations safe. Liver Transpl 2006;12:920-7.

2. Marcos A, Orloff M, Mieles L, Olzinski A, Sitzmann J. Reconstruction of double hepatic arterial and portal venous branches for right-lobe living donor liver transplantation. Liver Transpl 2001;7:673-9.

3. Thayer WP, Claridge JA, Pelletier SJ, Oh CK, Sanfey HA, Sawyer RG, et al. Portal vein reconstruction in right lobe living-donor liver transplantation. J Am Coll Surg 2002;194:96-8.

4. Lee SG, Hwang S, Kim KH, Ahn CS, Park KM, Lee YJ, et al. Approach to anatomic variations of the graft portal vein in right lobe living-donor liver transplantation. Transplantation 2003;75(3 Suppl):S28-32.

5. Hwang S, Lee SG, Ahn CS, Kim KH, Moon DB, Ha TY, et al. Technique and outcome of autologous portal $\mathrm{Y}$-graft interposition for anomalous right portal veins in living donor liver transplantation. Liver Transpl 2009;15:427-34.

6. Lee HJ, Hwang S, Ahn CS, Kim KH, Moon DB, Ha TY, et al. Long-term outcomes of portal Y-graft interposition for anomalous right portal veins in living donor liver transplantation. Transplant Proc 2012;44:454-6.

7. Ha TY, Hwang S, Moon DB, Ahn CS, Kim KH, Song $\mathrm{GW}$, et al. Conjoined unification venoplasty for graft double portal vein branches as a modification of autologous Y-graft interposition. Liver Transpl 2015;21:707-10.

8. Hwang S, Ha TY, Song GW, Jung DH, Moon DB, Ahn $\mathrm{CS}$, et al. Conjoined unification venoplasty for double portal vein branches of right liver graft: 1-year experience at a high-volume living donor liver transplantation center. J Gastrointest Surg 2016;20:199-205.
9. Kwon JH, Hwang S, Song GW, Moon DB, Park GC, $\mathrm{Kim} \mathrm{SH}$, et al. Conjoined unification venoplasty for triple portal vein branches of right liver graft: a case report and technical refinement. Korean J Hepatobiliary Pancreat Surg 2016;20:61-5.

10. Moon DB, Lee SG, Ahn C, Hwang S, Kim KH, Ha T, et al. Application of intraoperative cine-portogram to detect spontaneous portosystemic collaterals missed by intraoperative doppler exam in adult living donor liver transplantation. Liver Transpl 2007;13:1279-84.

11. Hwang S, Ahn CS, Kim KH, Moon DB, Ha TY, Song $\mathrm{GW}$, et al. Standardization of modified right lobe grafts to minimize vascular outflow complications for adult living donor liver transplantation. Transplant Proc 2012;44: 457-9.

12. Hwang S, Ha TY, Ahn CS, Moon DB, Kim KH, Song $\mathrm{GW}$, et al. Standardized surgical techniques for adult living donor liver transplantation using a modified right lobe graft: a video presentation from bench to reperfusion. Korean J Hepatobiliary Pancreat Surg 2016;20:97-101.

13. Hwang S, Lee SG, Ahn CS, Moon DB, Kim KH, Ha TY, et al. Outflow vein reconstruction of extended right lobe graft using quilt venoplasty technique. Liver Transpl 2006;12:156-8.

14. Hwang S, Ha TY, Ahn CS, Moon DB, Song GW, Kim $\mathrm{KH}$, et al. Hemodynamics-compliant reconstruction of the right hepatic vein for adult living donor liver transplantation with a right liver graft. Liver Transpl 2012; 18:858-66.

15. Sugawara Y, Makuuchi M, Tamura S, Matsui Y, Kaneko J, Hasegawa K, et al. Portal vein reconstruction in adult living donor liver transplantation using cryopreserved vein grafts. Liver Transpl 2006;12:1233-6. 